\title{
Real-time programmable acoustooptic synthetic aperture radar processor
}

\author{
Michael Haney and Demetri Psaltis
}

\begin{abstract}
The acoustooptic time-and-space integrating approach to real-time synthetic aperture radar (SAR) processing is reviewed, and novel hybrid optical/electronic techniques, which generalize the basic architecture, are described. The generalized architecture is programmable and has the ability to compensate continuously for range migration changes in the parameters of the radar/target geometry and anomalous platform motion. The new architecture is applicable to the spotlight mode of SAR, particularly for applications in which realtime onboard processing is required.
\end{abstract}

\section{Introduction}

Synthetic aperture radar (SAR) ${ }^{1-3}$ has become an increasingly important imaging technique. The significance of SAR stems from its ability to combine high angular resolution, otherwise available only with shorter wavelength real-aperture sensors, with the long-range and weather penetrating features of active radars. However, unlike real-aperture systems, the unfocused data collected with SAR systems must be first collected and stored, then focused through a computationally intensive 2-D integral operation. As the SAR's application domain has expanded, the computational requirements have increased, and the data processing techniques have by necessity become more sophisticated.

Optical signal processing (OSP) techniques have been applied to the collection and processing of SAR data since the introduction of the technique, and SAR remains the most successful application of optical information processing to date. The reasons OSP techniques apply so well to SAR stem from the analogy between the coherently detected radar echoes received from the object field in a SAR scenario and the interferometrically detected Fresnel diffraction pattern of a diffusive object illuminated with a coherent light

Michael Haney is with BDM Corporation, 7915 Jones Branch Drive, McLean, Virginia 22102, and Demetri Psaltis is with California Institute of Technology, Department of Electrical Engineering, Pasadena, California 91125.

Received 13 August 1987.

0003-6935/88/091786-11\$02.00/0.

(C) 1988 Optical Society of America. source. Synthesizing an image of a single-point scatter in the target scene from SAR signals is equivalent to the reconstruction of an image of a point source from an optical Fresnel hologram. The ability to format the SAR returns in a 2-D raster and record them on photographic film permits this analogy to be physically realized. The use of film as a high-density high-speed storage medium has provided an elegant solution to the collection and storage problems posed by the high data rates of the SAR returns. Indeed, film provides the means to exploit fully the 2-D parallelism and high interconnectivity afforded by optics.

For real-time SAR applications, however, the use of optical techniques has generally been precluded by the need for chemical processing of the film on which the radar data are recorded. This fact and the dramatic advances in integrated circuit technology have led to the use of electronic signal processing techniques in most existing real-time SAR processors. However, for some proposed real-time SAR applications, it is desirable to collect, store, and process the unfocused data onboard a small, and possibly unmanned, radar platform. Furthermore, to maintain high resolution, the onboard processor must have the flexibility to adapt its filtering characteristics to account for programmed or anomalous changes in the parameters of the radar/ target geometry. When combined with the size and power consumption constraints imposed by the radar platform, the data processing speed and flexibility requirements may be beyond the scope of electronic technology alone.

Recent advances in optical transducer technology, however, have given rise to renewed interest in realtime optical SAR techniques. ${ }^{4,5}$ In one approach a time-and-space integrating (TSI) architecture has been developed that uses acoustooptic (AO) Bragg 
cells and charge-coupled device (CCD) detector arrays to generate SAR images at real-time rates. ${ }^{6-8}$ This architecture compares favorably to the all-electronic approaches in the areas of speed, size, power consumption, and EMI/EMP immunity. We review the TSI approach and describe new hybrid optical/electronic techniques that generalize it by incorporating electronic programmability. The new architecture has the flexibility necessary to update its filter characteristics at real-time rates for motion compensation or programmed changes in the data collection geometry, yet it can be made small in size and low in power consumption.

In Sec. II we qualitatively review the SAR concept and data collection geometries. The SAR signals and signal processing requirements are then described in Sec. III to establish the formulation and notation used throughout this paper. Section IV contains a detailed description of the TSI approach and results of a demonstration of the approach with simulated SAR return signals. Finally, in Sec. V we describe the modifications needed to add programmability to the architecture and discuss the applicability of this architecture to spot light ${ }^{9,10}$ imaging modes. Proof-of-principle results of the programmable azimuth filter are presented. Furthermore, the approach is shown to have sufficient flexibility to compensate for the effects of range migration, range/azimuth coupling, and changes in the parameters of the radar/target geometry.

\section{SAR Data Collection Geometries}

The typical SAR data collection geometry is depicted in Fig. 1. The radar platform moves parallel to the ground at a constant velocity. The radar beam illuminates a portion of the target scene to one side of the flight path. A periodic pulse train is transmitted, and the echoes are received by the radar at different locations along the trajectory. The received signals are stored and processed using correlation techniques to generate an image. The coordinate axes of the SAR image are the range and azimuth positions of the point scatterers referenced to the radar's location at a particular point in time. A scatterer's range is determined by the round-trip delay of the radar pulses. High range resolution is achieved by using high-bandwidth coding of the transmitted waveform followed by pulse compression of each return. Each scatterer's azimuth position is determined by the phase history of the returns. High azimuth resolution is achieved by synthesizing a large aperture as the platform moves and performing a pulse compression operation on the phase history of the sequence of returns.

In strip-map SAR the antenna is fixed to the body of the platform so that a long swath of the target scene is illuminated as the platform moves. In spotlight mode SAR the beam is steered to maintain illumination on a specific region. In general, the spotlight mode can achieve higher azimuth resolution because the integration time is not determined by the size of the antenna footprint, as is the case in strip-map SAR. However, the longer integration times of the spotlight mode SAR

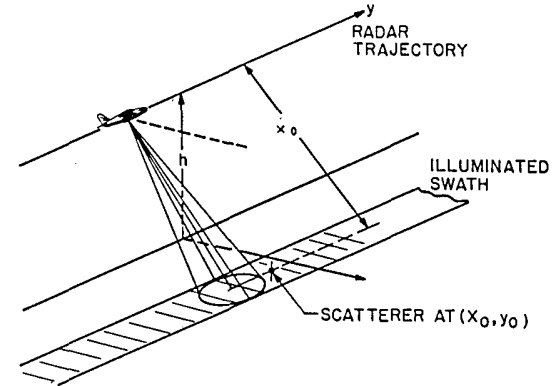

Fig. 1. Synthetic aperture radar data collection geometry.

cause the effects of range migration to be more pronounced. Range migration is the apparent movement of the target scatterers through range resolution cells due to the change in the distance between a target point and the radar during the data collection period. If range migration is non-negligible, i.e., the overall relative motion is greater than one resolution cell, the real-time processor must compensate for it to form a well-resolved image.

Finally, we distinguish the side-looking data collection geometry, in which the target is located directly abeam of the trajectory, from those in which there is a forward-looking aspect. In the side-looking aspect the squint angle, which is the angle between the radar's velocity vector and the antenna's pointing vector, is $90^{\circ}$. In this case the signal processing requirements are simpler because typically the range variation during the data collection period can be neglected. In the forward-looking geometry, however, the squint angle is $<90^{\circ}$, and the range decreases monotonically during the data collection. The signal processor must compensate for this range walk effect to produce sharp images.

\section{SAR Signal Processing}

Consider a radar that is carried on a platform moving on a straight trajectory parallel to the ground with velocity $v_{r}$ and altitude $h$. The antenna is pointed to the side of the flight path in the side-looking aspect and illuminates a strip on the ground as shown in Fig. 1. For simplicity we ignore the effects of the shape of the radar beam's footprint and assume that each scatterer in the swath is illuminated by a total of $N$ radar pulses as the footprint passes over it. The time period between pulses is $T$; thus the total data integration time is $N T$, and the size of the synthetic aperture is $v_{r} N T$. We assume that each radar pulse has pulse width $\tau$ with $\tau<T$ and is linear frequency modulated (LFM) with chirp rate $b$. The resulting transmitted waveform is a periodic pulse train given by

$$
s(t)=\sum_{n=0}^{N} \operatorname{rect}\left[\frac{t-n T}{\tau}\right] \exp \left[j \pi b(t-n T)^{2}\right] \exp (j \omega t),
$$

where $\omega$ is the carrier frequency of the radar.

Since the SAR imaging process is linear, it is completely characterized by its impulse response. This allows us to describe the imaging problem by consider- 
ing a single point-scatterer. Without loss of generality, we define the position of a scatterer by its projection onto the plane defined by the radar velocity vector and antenna pointing vector. This simplifies the notation somewhat. However, in the final image, the range coordinate must be scaled by the secant of the angle between this plane and the ground. The origin is defined by the position of the radar at $t=0$. For a scatterer located at $\left(x_{0}, y_{0}\right)$ the distance between it and the radar is given by

$$
R(t)=\left[x_{0}^{2}+\left(y_{0}-v_{r} t\right)^{2}\right]^{1 / 2} .
$$

If $x_{0} \gg v_{r} N T$, then

$$
R(t) \simeq x_{0}+\frac{\left(y_{0}-v_{r} t\right)^{2}}{2 x_{0}} .
$$

For simplicity, we assume that the strength of the radar signal is uniform within the footprint. The echo signal received at the radar is then proportional to $s(t-2 R(t) / c)$, where $c$ is the speed of light. From Eqs. (1) and (3) we may write an approximate expression for the signal received from the point scatterer:

$$
\begin{aligned}
r(t) \simeq & \sum_{n=0}^{N} \operatorname{rect}\left[\frac{t-\frac{2 x_{0}}{c}-n T}{\tau}\right] \\
& \times \exp \left[j \pi b\left(t-\frac{2 x_{0}}{c}-n T\right)^{2}\right] \exp \left[-j \omega\left(y_{0}-v_{r} n T\right)^{2} / x_{0} c\right] .
\end{aligned}
$$

In Eq. (4) we assume that the effects of range migration are negligible. (We show in Sec. $V$ how to handle them in our architecture if they are not negligible.) This permits us to approximate $R(t)$ by $x_{0}$ in the first two terms, which represent the time-delayed LFM waveform. We have also dropped an inconsequential constant phase term and the radar rf carrier term. In the third term of Eq. (4) the time-varying portion of the range delay is not negligible because it is multiplied by the high frequency $\omega$. Moreover, it is assumed that the temporal variation of the phase modulation within a single-pulse duration is negligible; this permits us to use the approximation $t \simeq n T$ in the third term. These approximations provide the basis for the SAR technique. The range position $x_{0}$ is estimated from the round-trip delay of the LFM waveform using pulse compression techniques, whereas the third term in Eq. (4) provides the means to estimate the azimuth coordinate $y_{0}$ of the scatterer. This term is quadratically phase modulated in the discrete time variable $n T$, and pulse compression techniques can be applied to it to achieve an estimate of the midpoint of this slow modulation, which corresponds to the azimuth coordinate $y_{0}$.

Although $r(t)$ is a 1-D function of time, it is convenient to think of it as a 2-D function of fast and slow time variables. We define the fast time to be $t_{1}=t-$ $n T$ in the first two terms of Eq. (4) and the slow time to be $t_{2}=n T$ in the third term. With these definitions we rewrite Eq. (4):

$$
\begin{aligned}
f\left(t_{1}, t_{2}\right)= & \operatorname{rect}\left(\frac{t_{1}-\frac{2 x_{0}}{c}}{\tau}\right) \exp \left[j b_{1}\left(t-\frac{2 x_{0}}{c}\right)^{2}\right] \\
& \times \operatorname{rect}\left[\frac{t_{2}-y_{0} / v_{r}-N T / 2}{N T}\right] \exp \left[j \frac{b_{2}}{x_{0}}\left(t_{2}-y_{0} / v_{r}\right)^{2}\right] .
\end{aligned}
$$

Equation (5) describes a 2-D asymmetrical Fresnel zone plate corresponding to the unfocused image of the scatterer. The required focusing is described by the following linear operation:

where

$$
g(x, y)=\sum_{t_{2}} \int f\left(t_{1}, t_{2}\right) h\left(t_{1}-\alpha x, t_{2}-\beta y, x_{0}\right) d t_{1},
$$

$$
\begin{aligned}
h\left(t_{1}, t_{2}, x_{0}\right)= & \operatorname{rect}\left(\frac{t_{1}}{\tau}\right) \exp \left(-j b_{1} t_{1}^{2}\right) \\
& \times \operatorname{rect}\left(\frac{t_{2}-\frac{N T}{2}}{N T}\right) \exp \left(-j b_{2} t_{2}^{2} / x_{0}\right),
\end{aligned}
$$

and $\alpha$ and $\beta$ are suitable constants. This linear operation is nearly shift invariant, except that the kernel is scaled in the azimuth coordinate $t_{2}$ by the range $x_{0}$. This effect is called range/azimuth coupling. In conventional nonreal-time optical SAR processing, the signal of Eq. (5) is detected, placed on a bias, and recorded on photographic film. The focused image is then obtained by 2-D Fresnel diffraction aided by anamorphic lenses that correct for range/azimuth coupling. However, examination of Eq. (7) reveals that the kernel required for focusing is separable in the two variables of integration. Since the data are in reality received one line at a time the separability suggests that focusing does not have to be implemented with a 2-D operation, as in the classical optical SAR processor, but instead can be accomplished with a cascade of two 1-D operations, thereby eliminating the need for a 2-D input transducer. It is this feature which is exploited in our approach as described in the following section.

\section{Time-and-Space Integrating Approach}

\section{A. Range Compression}

Rearranging Eq. (6), we have

$$
\begin{aligned}
g(x, y)= & \sum_{t_{2}=-N T / 2}^{N T / 2} \exp \left[j \frac{b_{2}}{x_{0}}\left(t_{2}-\beta y\right)^{2}\right] \\
& \times \int_{-\tau / 2}^{+\tau / 2} f\left(t_{1}, t_{2}\right) \exp \left[j b_{1}\left(t_{1}-\alpha x\right)^{2}\right] d t_{1} .
\end{aligned}
$$

Examination of Eq. (8) suggests that it is possible to implement the 2-D integral by first carrying out the integration over $t_{1}$ for each value of $t_{2}$ and all $x$. The acoustooptic implementation of this operation is shown schematically in Fig. 2. The received signal, after being mixed to the appropriate center frequency, is applied to the piezoelectric transducer of the acous- 


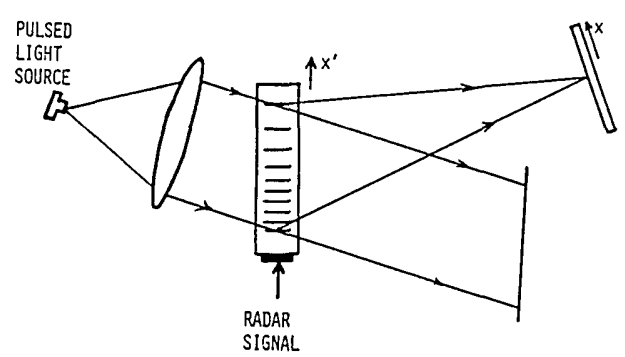

Fig. 2. Space integrating acoustooptic range focusing technique.

tooptic device (AOD). An acoustic wave is generated in the crystal that forms a moving 1-D diffraction grating whose transmittance is proportional to $r\left(t-x^{\prime}\right)$ $v_{a}$ ), where $x^{\prime}$ is the spatial coordinate along the direction of acoustic propagation in the crystal. At time instances $t=m T$, a collimated light source is pulsed with a pulse width that is less than the inverse of the bandwidth of the signal. This acts to freeze the moving diffraction grating. With the assumption that $\tau<$ $T_{a}<T$, the temporally sampled spatial representation of $r(t)$ in the AOD is strictly equivalent to its representation as a 2-D signal $f\left(t_{1}, t_{2}\right)$ with $t_{1}=x^{\prime} / v_{a}$. The integration over the range variable $t_{1}$ is, therefore, accomplished by an integration over the spatial variable $x^{\prime}$. With the assumption that the AOD is operated in the Bragg regime, only one diffraction order results. The induced diffraction grating due to the radar echo acts as a 1-D Fresnel lens which diffracts the collimated beam into either a diverging or converging cylindrical wave. Figure 2 shows the converging case. The propagation of light through a distance $z$ in free space is described by an integral identical in form to the integral over $t_{1}$ in Eq. (8). The light focuses at a distance $z=\pi v_{a}^{2} / \lambda b_{1}$ away from the AOD. The position of the focused spot in the transverse direction is determined by the position of the chirp in the AOD, which in turn is determined by the range to the scatterer. The light distribution at the plane where the light comes to a focus represents the SAR image focused in range only. Imaging along the second (azimuth) dimension is performed with a temporal integration of light over multiple radar pulses. This technique is described in the next section.

\section{B. Azimuth Compression}

The information necessary for focusing in the azimuth direction is contained in the phase of the range compressed image of the point scatterer. This image is a complex quantity with a magnitude that remains constant from pulse to pulse but with a phase that changes quadratically with $n$. Azimuth compression is achieved by temporally integrating this phase information on a detector array. Since detectors respond only to light intensity, we must interferometrically detect the azimuth phase history. Figure 3 depicts a common path interferometric scheme for azimuth phase detection, which is insensitive to mechanical vibration. The reference beam is derived from a sinusoidal signal that is added to the radar echo before it

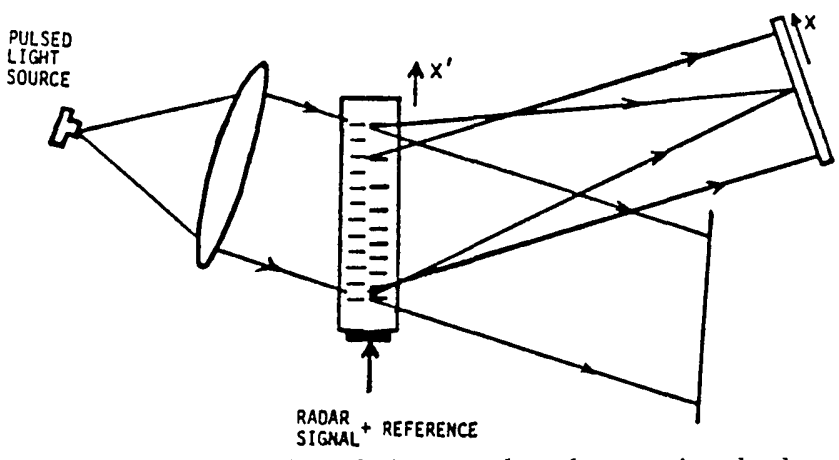

Fig. 3. Interferometric technique used to detect azimuth phase histories.

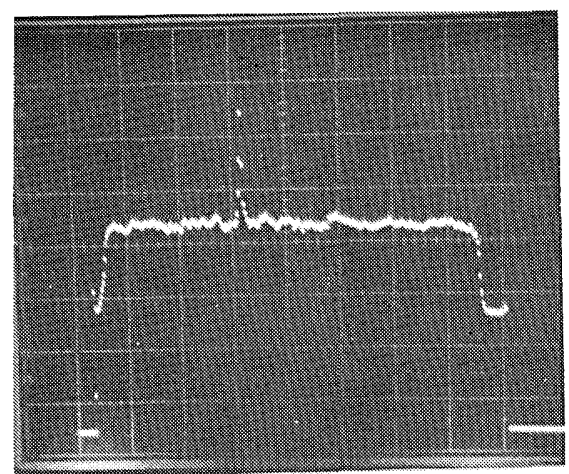

a

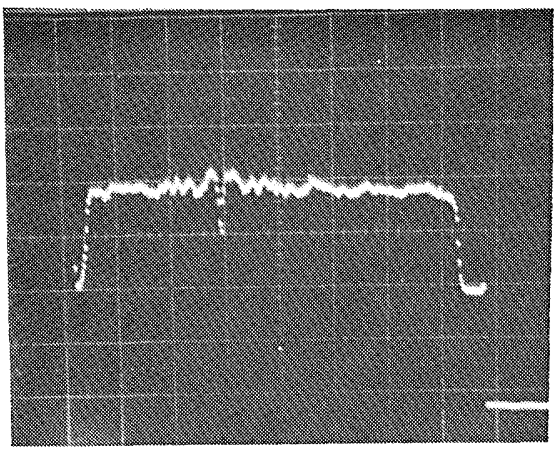

b

Fig. 4. CCD output showing range-dimension signals: (a) range focused signal and uniform reference; (b) same as (a) but with relative phase adjusted for destructive interference.

is applied to the AOD. The reference signal diffracts a plane wave that interferes with the range focused beam at the detector plane for all range bins. The detected light intensity for any point scatterer thus contains a term that is proportional to the real part of the third term in Eq. (5). As the phase changes with $n$, the intensity seen by the detector varies from constructive to destructive interference accordingly. Examples of range focused data, whose phases have been detected with this technique, are shown in Fig. 4.

The signal detected at the output of the interferometer of Fig. 3 is the result of the integration in the variable $t_{1}$ in Eq. (8), with the range variable $x$ as the 
output spatial coordinate. The azimuth output coordinate $y$ is obtained by expanding the dimension perpendicular to $x$, as shown in the schematic of the full system in Fig. 5. The collimated beam from the pulsed laser diode is focused into the AOD by a cylindrical lens. The diffracted light is recollimated in the $y$ direction by a second cylindrical lens, and in the $x$ direction the rays follow paths as depicted in Fig. 3. (The blocked undiffracted beam is not shown in Fig. 5.) As a result, the range focused image is spread vertically, as shown in Fig. 5. A mask, whose intensity transmittance function $t_{1}(x, y)$ consists of a bias term and the real part of the $y$-dependent portion of the complex kernel in Eq. (8), is positioned directly in front of the CCD array. The intensity transmittance of this mask is a LFM signal whose chirp rate varies with range to account for range/azimuth coupling:

$$
t_{1}(x, y)=1 / 2+1 / 2 \cos \left[b_{2}(\beta y)^{2} / x\right] .
$$

An example of this mask is shown in Fig. 6; the range coordinate is along the horizontal direction. Processing with this mask produces either the real or imaginary component of the SAR image by detecting either the $I$ or $Q$ component of the return signals. Several techniques exist to obtain the full complex image, ${ }^{6}$ including using a second mask that is in quadrature with the first. For purposes of this discussion we proceed with the description of the architecture and omit the details of the complex representation.

If $I_{0}\left(x, t_{2}\right)$ is the light intensity incident at the output plane in Fig. 5, the light detected by the CCD array is $I\left(x, y, t_{2}\right)=I_{0}\left(x, t_{2}\right) t_{1}(x, y)$. The CCD array is operated in the time-delay-and-integrate (TDI) mode along the $y$ direction during the integration of $N$ pulses. This means that the photogenerated charge is continuously transferred along the azimuth direction during exposure. The motion of the charge relative to the stationary reference mask performs a correlation of the mask function with the temporally modulated light intensity. After $N$ light pulses, the accumulated charge pattern on the CCD array is given by

$$
\begin{aligned}
I_{C C D}(x, y) & =\sum_{t_{2}=-N T / 2}^{N T / 2} I\left(x, y+v_{c} t_{2}, t_{2}\right) \\
& =\sum_{t_{2}=-N T / 2}^{N T / 2} I_{0}\left(x, t_{2}\right) t_{1}\left(x, y+v_{c} t_{2}\right),
\end{aligned}
$$

where $v_{c}$, the effective velocity of the moving charge, is given by $v_{c}=\delta y / T$ and $\delta y$ is the pixel separation on the CCD. Since the mask function has been chosen to match the azimuth phase history, the resulting correlation in the $y$ direction is actually an autocorrelation, which results in a sharply focused signal in the $y$ direction, as desired.

\section{Output Image}

A detailed analysis, which includes all the bias components that arise from the interferometric detection and the mask, shows that $I_{\mathrm{CCD}}(x, y)$ consists of the real (or imaginary) component of $g(x, y)$, which is the de-

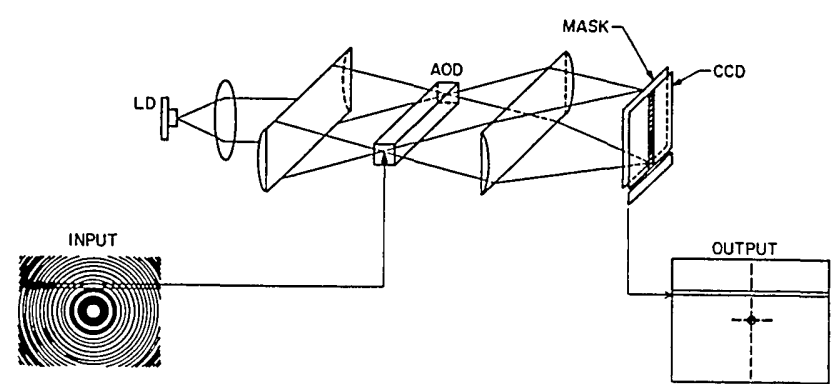

Fig. 5. Real-time acoustooptic SAR processor.

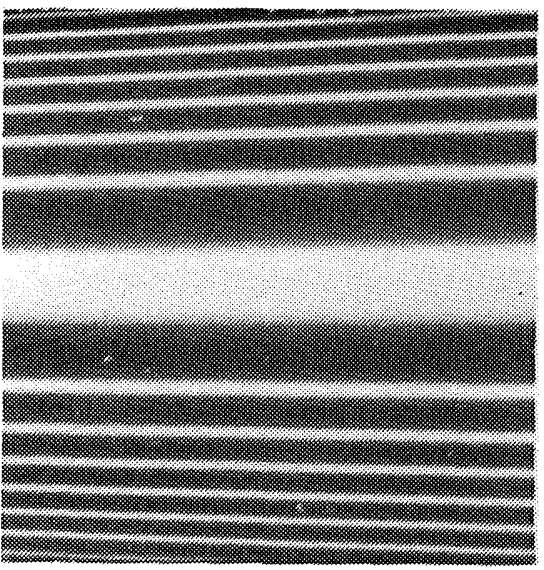

Fig. 6. Example of a reference mask.

sired image, and a constant bias. ${ }^{4}$ An isometrically displayed image of a simulated point scatterer focused in real-time without removal of the bias is shown in Fig. 7(a). The sharply focused image is evident atop a bias ridge that is contained in the range bin of the scatterer and is due to the interferometric detection scheme. The plateau on which the bias ridge and the signal are sitting is formed by the approximately uniform reference beam. Various techniques are available for removing the unwanted bias components in real-time to provide a high-quality image. ${ }^{7}$ Figure 7 (b) shows the results of one bias removal technique in which the real and imaginary components of the simulated target have been combined to form a complete image of the scatterer.

\section{Generalization of the Architecture}

We have assumed thus far a side-looking geometry, in which the parameters of the problem are fixed and range migration is negligible. However, in some SAR applications the parameters of the radar/target geometry may change dynamically, and range migration cannot be ignored. This is the case, for example, in a forward-looking spotlight mode geometry in which parameters such as the radar velocity and initial squint angle of the radar beam must be variable.

Furthermore, the effects of anomalous motion of the radar platform may degrade the resolution and the filtering characteristics of the real-time imager unless it is capable of compensating for this. Clearly the TSI must be programmable to handle the general situation. 


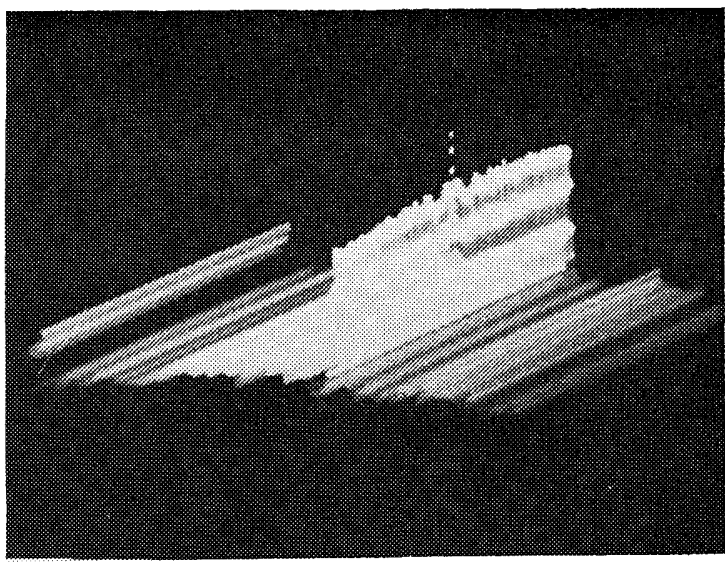

a

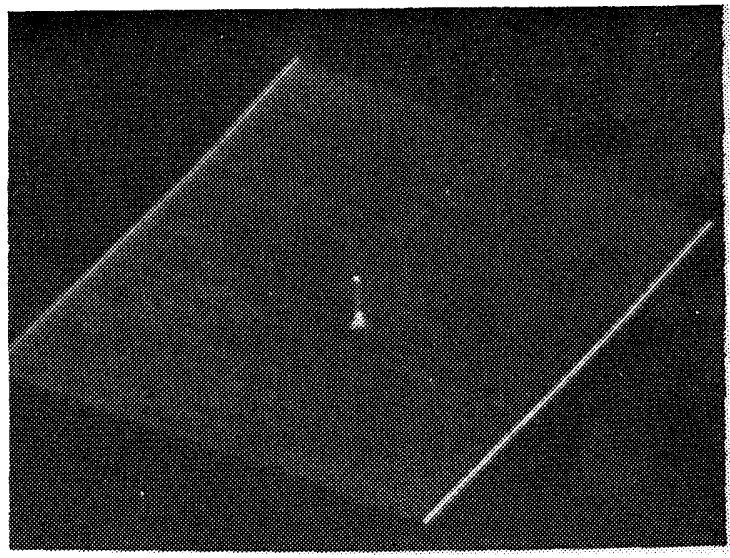

b

Fig. 7. Isometric displays of real-time processor outputs for simulated point scatterer input: (a) with bias terms; (b) without bias terms.

To accomplish this with the system of Fig. 5 the mask could be replaced by a real-time 2-D spatial light modulator (SLM) on which the updated filter function could be written. However, as we show in the next sections, this is unnecessary.

\section{A. Interferometrically Generated Reference Function}

Examination of the 2-D mask reference function in Fig. 6 reveals that it is approximated well by a 1-D baseband LFM signal in the azimuth direction whose scale varies in the range direction to account for range/ azimuth coupling. This approximation is valid because it is assumed that the overall range to the target scene is large when compared to the width of the strip that is being imaged. This suggests that a 1-D SLM can be used to enter the reference function in the processor in conjunction with an anamorphic lens arrangement to provide the scaling in the orthogonal direction. Figure 8 is a schematic diagram of a specific implementation of this idea.

An electronic signal, which consists of a chirp summed to a sinusoidal reference, is applied to an AOD. After the acoustic waves have propagated to the center of the AOD, the laser diode is pulsed to

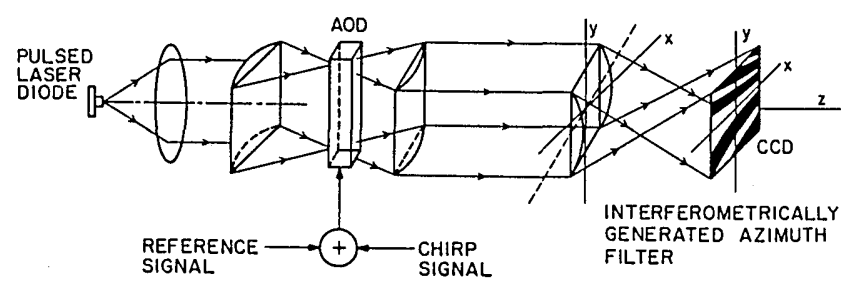

Fig. 8. Optical setup for the interferometric generation of the SAR azimuth filter.

freeze the moving diffraction grating in a manner identical to the way the radar signal is sampled in the AOD of the SAR architecture described previously. An anamorphic lens arrangement is used to spread the diffracted light uniformly in the $x$ direction at the detector plane. The undiffracted light (not shown in Fig. 8) is blocked and not used. In the $y$ direction, the wave due to the sinusoid is a plane wave that is transformed into a cylindrical wave at the detector plane. The diffracted light due to the chirp signal is a cylindrical wave that is transformed into a cylindrical wave of different curvature at the detector plane. The two waves have different radii of curvature at the detector plane. Under the paraxial approximation, the resulting interference pattern is a LFM signal in the $y$ direction. The scaling needed for range/azimuth coupling compensation is accomplished by tilting a cylindrical lens about the $y$ axis, as shown.

An architecture employing the interferometrically generated reference function has the flexibility to adapt to changes in the radar/target geometry by changing the characteristics of the electronically generated chirp. The parameters that may be changed include the starting frequency, starting time, and chirp rate of the azimuth reference signal. In the following section we describe one such architecture.

\section{B. Programmable Architecture}

To synthesize a programmable real-time SAR architecture, we integrate the interferometric azimuth filter technique with the range processing technique of Fig. 2. This could be accomplished with an additive interferometric architecture in which the AOD containing the radar signal is placed in one arm of a MachZehnder interferometer, and the AOD containing the azimuth reference is oriented orthogonally to the first AOD and placed in the second arm. An alternate and generally preferred solution is a multiplicative interferometric approach in which the two systems are cascaded. The cascaded system is shown schematically in Fig. 9. The top view shows the path of the light beams in the range dimension. The light diffracted by the induced 1-D Fresnel zone plate focuses on the CCD to perform the range compression. As in the original architecture, this wave interferes with a beam that is collimated in the $x$ direction and is due to the sinusoidal reference that is added to the signal applied to the first AOD. Both beams pass through the second AOD containing the azimuth filter signal and a second sinusoidal reference. The sum of signals corresponding to 

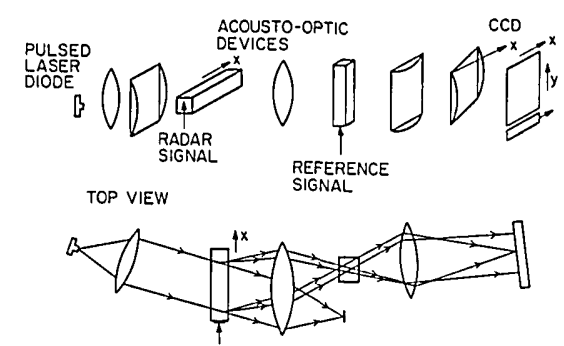

SIDE VIEW

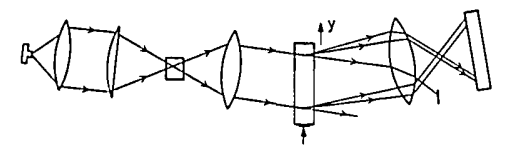

Fig. 9. Optical architecture for SAR imaging with the azimuth reference introduced through a second AO cell.

the azimuth kernel and a sinusoidal reference is periodically applied to the second AOD in synchronism with the radar pulse repetition frequency (prf). As in the original architecture, the range focused signal is detected interferometrically to preserve its phase. In this case, however, the light that is diffracted by the first AOD is rediffracted by the azimuth signal in the second AOD. This doubly diffracted beam thus contains the product of the range focused signal and the appropriate azimuth reference. Similarly, the reference signal from the first AOD is rediffracted by the reference signal in the second AOD to provide a reference beam at the output plane. The two doubly diffracted beams interfere at the detector plane to produce an intensity pattern that contains a term proportional to the correct range focused signal and the azimuth filter, precisely as in the architecture with the fixed mask. Temporal integration on a scrolling CCD detector produces the final focused SAR image as before.

A proof-of-principle demonstration of the programmable architecture of Fig. 9 was conducted. A fixed LFM signal that was generated by a surface acoustic wave (SAW) chirp generator was used as the azimuth filter. The azimuth filter characteristics were adjusted by varying the optical magnification along the $y$ direction. Simulated single scatterer signals were generated as before. Figure 10 shows an isometrically displayed image of a simulated scatterer generated with this experimental arrangement. A comparison of Figs. 7(a) and 10 shows that the operation of the multiplicative architecture, with an electronically generated azimuth reference, is essentially identical to that of the original fixed mask processor.

The novelty of this approach stems from the flexibility made possible by its electronic degree of freedom. There are four electronic-optical interfaces in this architecture, one for each optical transducer in Fig. 9. These interfaces are highlighted in the simplified schematic diagram of the architecture shown in Fig. 11. In the following discussion we show how, taken together, these interfaces provide the means to handle spotlight mode geometries and to compensate simultaneously

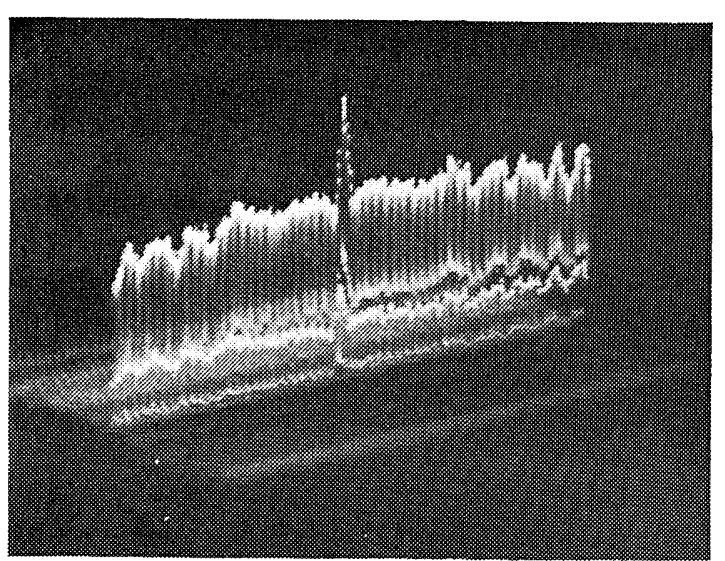

Fig. 10. Output of the processor with an electronically generated azimuth reference filter.

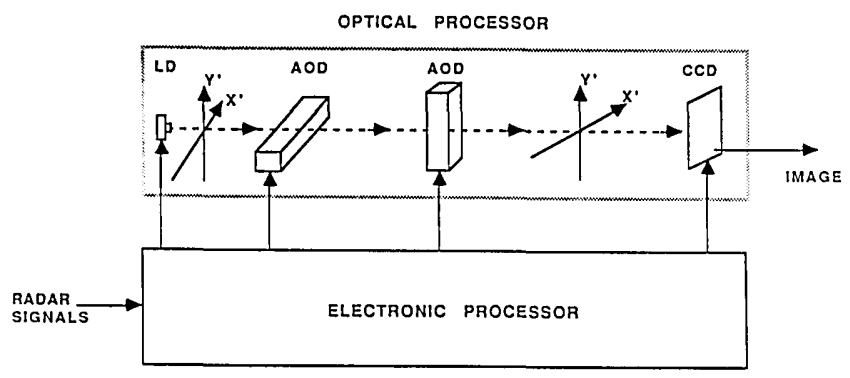

Fig. 11. Hybrid processor interfaces.

for the effects of range migration, range/azimuth coupling, and dynamic changes in the data collection geometry.

The first important feature of this architecture is that azimuth focusing can be performed without shifting the charge on the CCD array. Instead the azimuth kernel in the AOD can be progressively shifted in the $y$ direction as a function of the slow time variable $t_{2}$. This is accomplished by generating the azimuth kernel at a slightly higher prf than that of the radar and laser diode. As a result, the acoustic signals in the second AOD, seen by two consecutive laser pulses, are shifted versions of one another. This effect is entirely analogous to shifting the photogenerated charge in the CCD array and can, therefore, be used to perform azimuth compression. This method produces a framed, rather than a scrolled, output on the CCD. The technique is, therefore, more appropriate for spotlight mode SAR applications in which a framed output is desired.

Range walk is compensated in this architecture by electronically adjusting the timing of the laser pulse to keep the range focused signals in the same range bin throughout the integration period. The amount of adjustment is determined by the gross change in the range as a function of time as determined by the parameters of the forward-looking geometry. The period between successive return pulses is reduced by a time increment $t^{\prime}$ for each pulse, where $t^{\prime}$ is given by 


$$
t^{\prime}=2 v_{r} T \cos (\theta) / c,
$$

where $\theta$ is the squint angle. As the parameters of the radar/target geometry change, $t^{\prime}$ can easily be changed to account for them.

The gross Doppler shift due to range walk is also compensated electronically with the aid of a programmable frequency synthesizer that generates the appropriate compensating frequency to mix with the radar return before it is applied to the $\mathrm{AO}$ cell. The compensating frequency is given by

$$
\omega_{d}=v_{r} \omega \cos (\theta) / c .
$$

Shifting the radar returns by $\omega_{d}$ ensures that the phase histories are at baseband and, therefore, are adequately sampled by the radar and laser diode pulse repetition frequency.

To compensate for range/azimuth coupling, a cylindrical lens can be tilted as shown in Fig. 9. However, because the range/azimuth coupling parameter varies as a function of range, it can also be compensated by modulating the reference function in the first AOD by a suitable phase function that is calculated in a digital processor from the parameters of the radar/target geometry. This phase function serves to make the effective quadratic azimuth phase histories have the same chirp rate in all range bins. Under the previously stated assumption that the desired azimuth phase function is scaled linearly in the range direction, we have the following form for the compensating phase function:

$$
f_{r / a}\left(x^{\prime}, n\right)=\exp \left(j b_{3} x^{\prime} n^{2}\right),
$$

where $b_{3}$ is a constant determined from the parameters of the SAR geometry and the optical processor, and $x^{\prime}$ is the range coordinate in the AOD corresponding to the difference between the range to a scatterer and the range bin at the center of the target scene in the spotlight geometry (i.e., $x^{\prime}=0$ for the range bin passing through the center of the target scene). This programmable solution to range/azimuth coupling gives the architecture the flexibility to adjust for changes in geometry without having to change the tilt of a lens or lenses, which may introduce distortions that are difficult to correct for all tilt angles.

\section{Application to Spotlight Mode SAR}

When long integration times are used to achieve high azimuth resolution, range curvature (which is a component of range migration that is approximately quadratic in time) becomes significant and must be dealt with. This is especially true for spotlight mode SARs, where integration periods may be arbitrarily long. Figure 12 depicts the spotlight mode geometry, when projected into the plane containing the radar velocity vector and radar pointing vector. The center of the region to be imaged is located at squint angle $\theta_{0}$ and range $R_{0}$ at $n T=0$. We assume that the radar pattern is steered to maintain illumination on the region of interest throughout the integration period (hence the name spotlight). The average spotlight

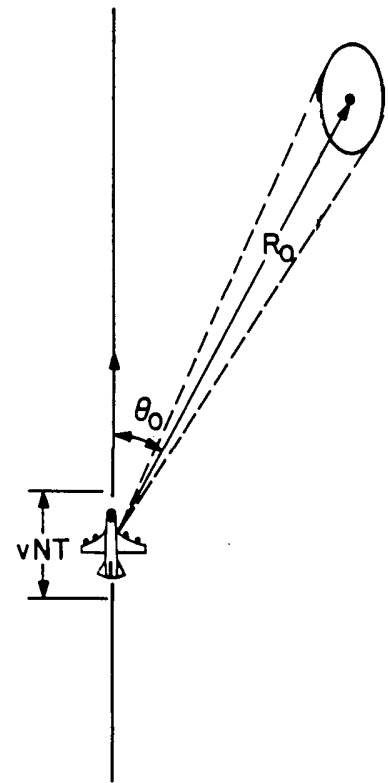

Fig. 12. Spotlight mode SAR geometry.

rate needed to accomplish this is $\dot{\theta}=v_{r} \sin \theta_{0} / R_{0}$. Data are collected for a period $t=-N T / 2$ to $t=N T / 2$. The range to the center point of the scene is given by

$$
r_{0}(n T)=R_{0} \sqrt{1-\frac{2 v_{r} n T \cos \theta_{0}}{R_{0}}+\frac{\left(v_{r} n T\right)^{2}}{R_{0}^{2}}} .
$$

We assume that $v_{r} N T \ll R_{0}$. Therefore, with the assumption that all higher-order terms are much less than the width of a range resolution element, keeping the first three terms of a series expansion of Eq. (14) yields

$$
r_{0}(n T) \simeq R_{0}-v_{r} n T \cos \theta_{0}+\frac{\left(v_{r} n T \sin \theta_{0}\right)^{2}}{2 R_{0}} .
$$

We define the location of an arbitrary point scatterer in the scene by its range and angular coordinates $(\rho, \gamma)$ with respect to $\left(R_{0}, \theta_{0}\right)$ at $t=0$. The position of a scatterer at $t=0$ is thus $\left(R_{0}+\rho, \theta_{0}+\gamma\right)$. The range to this scatterer at an arbitrary time is

$$
\begin{aligned}
r(n, \rho, \gamma)= & R_{0}+\rho-v_{r} n T \cos \left(\theta_{0}+\gamma\right) \\
& +\frac{\left(v_{r} n T \sin \left(\theta_{0}+\gamma\right)^{2}\right.}{2(r+\rho)} .
\end{aligned}
$$

We assume $\rho \ll R_{0}$ and $\gamma \ll \theta_{0}$. After expanding Eq. (16) in a series and keeping only those terms which are not negligible with respect to a radar wavelength, we have

$$
\begin{aligned}
r(n, \rho, \gamma) \simeq R_{0}+\rho-v_{r} n T \cos \theta_{0}+\left(v_{r} n T \sin \left(\theta_{0}\right) \gamma\right. \\
+\frac{\left(v_{r} n T \sin \theta_{0}\right)^{2}}{2 R_{0}}\left(1-\rho / R_{0}\right)+\frac{\left(v_{r} n T\right)^{2} \gamma \sin 2 \theta_{0}}{2 R_{0}} .
\end{aligned}
$$

\section{Range Processing}

For range processing we assume that all terms in Eq. (17) that are higher than quadratic in $\rho, \gamma$, and $n$ are 
negligible when compared to a range resolution cell in the processor. The first term $R_{0}$ is compensated by a simple fixed delay of $2 R_{0} / c$ in the pulsing of the laser diode in the processor. The third term corresponds to the overall range walk and is compensated by dithering the laser diode interpulse period, as described earlier. With these assumptions the remaining range delay term is

$$
r_{1}(n, \rho, \gamma)=\rho+\gamma v_{r} n T \sin \theta_{0}+\frac{\left(v_{r} n T \sin \theta_{0}\right)^{2}}{2 R_{0}}
$$

The third term in Eq. (18) corresponds to the component of range curvature that is common to all scatterers and can be compensated in the same manner as the range walk. The remaining range delay components for a scatterer at $(\rho, \gamma)$ is, therefore,

$$
r_{2}(n, \rho, \gamma)=\rho+\gamma v_{r} n T \sin \theta_{0} .
$$

Rewriting Eq. (19) in terms of the spotlight rate $\dot{\theta}$ yields

$$
r_{2}(n, \rho, \gamma)=\rho+\left(\gamma R_{0}\right) \dot{\theta} n T .
$$

The coordinate axes on the CCD array at the output of the processor are defined to be $(x, y)$, where the $x$ axis is aligned with the AO cell containing the radar signal. The image of a scatterer at $(\rho, \gamma)$ is defined to have desired coordinates on the CCD: $x=C_{1} \rho$ and $y=$ $C_{2} \gamma R_{0}$. If $C_{1}=C_{2}$, the image scaling on the CCD equals that of the actual scene in the plane defined in Fig. 12; we assume this to be the case in the processor. As shown in Eq. (20), the actual delay of the range focused signal at the output plane of the processor shifts as a linear function of time. For this case, the position of the range focused signal at the output plane is

$$
\bar{r}_{2}(n, x, y)=x+\dot{\theta} y n T .
$$

Examination of Eq. (21) reveals that, if the integration period is long enough, the range focused signal may move through several range resolution elements with time and result in blurring. However, if the CCD array is rotated during data collection at the spotlight angular rate about the optical axis with the optical axis defined to be $(0,0)$, at pixel $(x, y)$ the remaining timedependent range migration of Eq. (21) will be canceled out, yielding the desired constant range history:

$$
\bar{r}(n, x, y)=x .
$$

\section{Azimuth Processing}

The component of the phase of the return signals from a scatterer that varies with $n$ is given by

$$
\phi(n, \rho, \gamma)=-\frac{2 \omega}{c} r(n, \gamma, \rho) .
$$

After substitution of Eq. (17), dropping insignificant constant phase terms and assuming that the gross Doppler shift is compensated as described above, we have

$$
\begin{aligned}
\phi(n, \rho, \gamma)= & -\frac{2 \omega}{c}\left[\delta v_{r} n T \sin \theta_{0}+\frac{\left(v_{r} n T \sin \theta_{0}\right)^{2}}{2 R_{0}}\left(1-\rho / R_{0}\right)\right. \\
& \left.+\frac{\left(v_{r} n T\right)^{2} \gamma \sin 2 \theta_{0}}{2 R_{0}}\right] .
\end{aligned}
$$

The last term is a slowly varying function of $\gamma$ and $t$ only and, therefore, can be compensated by an appropriate programmed phase function in the AOD containing the azimuth filter. After completing the square and dropping the component that is constant with $t$, the phase function from a scatterer takes the following form:

$$
\phi(n, \rho, \gamma) \simeq \frac{-\omega}{R_{0} C}\left(1-\rho / R_{0}\right)\left[v_{r} n T \sin \theta_{0}+\left(R_{0}+\rho\right) \gamma\right]^{2} .
$$

This function is quadratic in time, has a center of curvature proportional to the azimuth position $\left[\gamma\left(R_{0}+\right.\right.$ $\rho)$ ] of the scatterer, and has a chirp parameter that varies linearly (under the stated assumptions) with the range component. This range dependence in the azimuth chirp rate is called range/azimuth coupling. For reasons that become clear shortly, let us assume that a phase component is added to the return signal which has the form

$$
\Delta \phi(n, \rho, \gamma)=\frac{\omega}{C R_{0}}\left(v_{r} n T \sin \theta_{0}\right)^{2} \rho / R_{0} .
$$

This can be accomplished in the processor, as described earlier, by adding a programmed phase function to the reference signal in the first AOD of the form given by Eq. (13). After adding this phase function to Eq. (24) and completing the square as before, the resulting effective phase history of the range focused signal at the output plane of the processor, for a scatterer at $(\rho, \gamma)$, is given by

$\phi_{\mathrm{eff}}(n, \rho, \gamma) \simeq \frac{-\omega}{R_{0} C}\left(1-\frac{2 \rho}{R_{0}}\right)\left[v_{r} n T \sin \theta_{0}+\left(R_{0}+2 \rho\right) \gamma\right]^{2}$.

To perform azimuth compression, using the autocorrelation technique described in Sec. III, the processor must generate a phase function with a range-dependent chirp parameter equal to that given by Eq. (27). To accomplish this we modulate the azimuth filter AOD with the following phase function

$$
\phi^{\prime}(n T, y)=\frac{-\omega_{0}}{C_{1}^{2} c R_{0}}\left(y+C_{1} v_{r} n T \sin \theta_{0}\right)^{2} .
$$

Since the CCD is rotating, the resulting phase function, as viewed by a pixel at range coordinate $x$ on the array, will be modified from that of Eq. (28) to yield

$$
\phi_{\mathrm{CCD}}^{\prime}(n T, y)=\frac{-\omega_{0}}{C_{1}^{2} c R_{0}}\left(y-\dot{\theta} x n T+C_{1} v_{r} n T \sin \theta_{0}\right)^{2} .
$$

With the previously stated assumptions that $x=C_{1} \rho$ and $y=C_{1} \gamma R_{0}$, Eq. (29) reduces to

$\phi_{\mathrm{CCD}}^{\prime}(n T, \rho, \gamma) \simeq \frac{-\omega_{0}}{C R_{0}}\left(1-\frac{2 \rho}{R_{0}}\right)\left[v_{r} n T \sin \theta_{0}+\left(R_{0}+\rho\right) \gamma\right]^{2}$.

This phase function has the same range-dependent chirp rate as Eq. (27) and, therefore, will result in 
proper azimuth focusing for each range bin, as desired. The final image that forms on the rotating CCD, therefore, will not be blurred by the effects of range migration or range/azimuth coupling.

\section{Output Image}

After an integration period that is long enough to achieve the desired azimuth resolution, the image is read off the CCD, the bias components are removed by one of the techniques described previously, ${ }^{7}$ and the image can be displayed. A new image of the same scene can then be formed, if desired, using updated values for $\theta_{0}$ and $R_{0}$.

We note that a difference exists between Eqs. (27) and (30) in the azimuth position of the center of curvature. This difference will cause the azimuth position of each focused scatterer on the CCD to be scaled by approximately $\left(1+\rho / R_{0}\right)$. This effect is analogous to the apparent scaling of objects when viewed visually that are extended in range, such as the parallel boarders of a long straight road viewed from a position on the road. This scaling can be ignored, or straightforward electronic postprocessing of the image can be used to adjust the scaling of the axes, if desired.

\section{Performance Limits}

In this section we briefly review the key performance characteristics of the TSI approach and discuss the factors in the architecture that determine their fundamental limits. Image resolution is limited chiefly by the array size of the CCD detector and/or the spacebandwidth product (SBWP) of the AODs used. CCD arrays with up to $2000 \times 2000$ pixels are now commercially available as well as AODs with (claimed) SBWPs of $>2000$. Therefore, image sizes approaching a resolution of $4 \times 10^{6}$ pixels are theoretically possible. However, to attain a resolution close to this limit, a direct subtraction bias removal technique ${ }^{7}$ must be employed that requires additional optics and another CCD array on which to calculate the bias components. This technique has been demonstrated. ${ }^{11}$ Other bias removal techniques involve the use of a carrier and envelope detection. The data in Fig. 7(b) were produced in this manner. Carrier techniques require the spot size on the array to be at least several pixels $(\sim 6)$ across, thereby reducing the achievable image size to $\sim 300 \times 300$.

The coherent optical detection scheme plays a fundamental role in determining the dynamic range of the output image. Since the detected signal is proportional to the amplitude of the point scatterer's return signal, the resulting time-integrated peak in Fig. $7(\mathrm{~b})$ is also proportional to the magnitude of this complex amplitude. Therefore, 100 distinguishable signal levels at the detector output correspond to a factor of $\sim 10,000$ in power (between the highest and lowest distinguishable scatterers in the displayed SAR image). The intrinsic dynamic range of currently available CCDs is of the order of several hundred. This can be improved by using cooling to reduce the dark cell noise. The advantage of coherent detection is offset somewhat by the need to allocate a large portion of the CCD dynamic range to the bias terms. The portion of the detector dynamic range that can be used for representing the SAR image depends on the characteristics of the SAR scene itself. Images of simple point scatterers can be generated with nearly half of the dynamic range of the CCD. For more complex scenes, the dynamic range drops as $1 / M^{1 / 2}$, where $M$ is the average number of equivalent scatterers in the same range bin within a distance equal to the size of the synthetic aperture in the azimuth direction.

The frame rate achievable with this architecture is determined by the frame rate of the CCD array. Most commercially available CCD arrays of moderate size $(\sim 500 \times 500)$ are capable of 30 frames/s, which is more than adequate for most SAR applications, where integration periods are of the order of seconds. In the original version of the architecture, in which the azimuth compression is achieved through the shifting of charge on the CCD array, the maximum prf achievable is limited to the CCD shift rate. However, in the programmable architecture, in which the shifting is done in the AOD, no such limit exists.

\section{Conclusion}

The programmable real-time TSI SAR architecture is an example of a hybrid optical/electronic signal processing approach that in many ways combines the best features of both worlds. All the electronically programmed signals required are straightforward to calculate and can be handled by currently available digital processors at real-time rates without taxing their capabilities. The coupling of electronic, optical, and mechanical transductions performs an interpolation of the focused data as it is being generated. This interpolation is necessary to maintain high resolution in the image when range curvature is not negligible. Performing the interpolation operation in the optical domain is computationally efficient. Conversely, it is well known that this interpolation operation is extremely difficult to do digitally at real-time rates; this is especially true if size and power are severely constrained. The effective distribution of the computational load between optics and electronics with this approach means that an actual embodiment of this SAR architecture is potentially compact $\left(<2000 \mathrm{~cm}^{3}\right)$ and has low-power requirements $(<50 \mathrm{~W})$ for both the digital and electrooptical sections.

The authors thank Kelvin Wagner, Jeffrey Yu, and Tom Bicknell for help in several aspects of this work and for many discussions on the subject. This work was supported by the Air Force Office of Scientific Research and General Dynamics.

\section{References}

1. L. Cutrona et al., "On the Application of Coherent Optical Techniques to Synthetic Aperture Radar," Proc. IEEE 54, 1026 (1966).

2. C. Sherwin, J. Ruina, and D. Rawcliffe, "Some Early Developments in Synthetic Aperture Radar Systems," IRE Trans. Mil. Electron. MIL-6, 111 (1962). 
3. L. Cutrona and G. Hall, "A Comparison of Techniques for Achieving Fine Azimuth Resolution," IRE Trans. Mil. Electron. MIL-6, 119 (1962).

4. D. Psaltis and K. Wagner, "Real-time Optical Synthetic Aperture Radar (SAR) Processor," Opt. Eng. 21, 822 (1982).

5. C. C. Aleksoff et al., "Optical Hybrid Backprojection Processing," Proc. Soc. Photo-Opt. Instrum. Eng. 422, 000 (Apr. 1983).

6. D. Psaltis, M. Haney, and K. Wagner, "Real-time Synthetic Aperture Radar Processing," in Proceedings, NASA Optical Information Processing Conference (Aug. 1983), p. 199.

7. M. Haney and D. Psaltis, "Acoustooptic Techniques for Real- time SAR Processing," Proc. Soc. Photo-Opt. Instrum. Eng. 545, (Apr. 1985).

8. D. Psaltis and M. Haney, "Real-time Synthetic Aperture Radar Processors," in Optical Signal Processing, J. Horner, Ed. (Academic, New York, 1987)

9. D. A. Ausherman et al., "Developments in Radar Imaging," IEEE Trans. Aerosp. Electron. Syst. AES-20, 323 (1984).

10. D. C. Munson et al., "A Tomographic Formulation of Spotlightmode Synthetic Aperture Radar," Proc. IEEE 7, 987 (1983).

11. K. T. Stalker et al., "Comparison of Real-time Acousto-optic SAR Processor Architectures," Proc. Soc. Photo-Opt. Instrum. Eng. 827, (1985).

\section{Documention Preserved: Report from the International Catalog of Sources for} History of Physics and Allied Sciences

This is our regular survey of archives and other repositories, giving information on materials of interest to historians and others not previously reported in this Newsletter. Many of these are new deposits not yet processed; others have restricted access. Please contact the repository for further information.

California Institute of Technology, Millikan Memorial Library, Institute Archives, Pasadena, CA (contact Judith Goodstein)

Papers of Gilbert Rusk Van Dyke III, 1943-1982. Physicist. Includes correspondence, research notes, bibliographies, and other material documenting the work and research of Scripps Institution of Oceanography graduate student Gilbert Rusk Van Dyke. Van Dyke was admitted to doctoral degree candidacy in 1980 and was conducting research on his dissertation under the direction of Seibert Quimby Duntley at the time of his death. The papers include material documenting his experiences as a Scripps graduate student. 1968-1982. 2.5 ln. ft.

Scripps Institution of Oceanography. Subject Files Collection. An artificial collection created by Scripps archivist Elizabeth Nobel Shor in 1976. Contains manuscript and archival material from many different sources filed under subject headings determined and established by Shor. Includes extensive information on Scripps financial affairs, Scripps sponsored research projects, Scripps expeditions, and Scripps research vessels. The bulk of the collection documents the period 1930-1970. 1903-1980. $36.25 \mathrm{ln}$. ft.

Papers of Joshua Irving Tracey, Jr., 1915- Geologist. Includes correspondence, memoranda, press releases, committee reports, goals, statements and other material generated or collected by Tracey concerning Project Mohole. The papers include material on the American Miscellaneous Society and the Long Cores Project. Correspondents include Harry Ladd, Gordon Lill, and Hollis D. Hedberg. 1957-1967. 75 ln. $\mathrm{ft}$.

University of Toronto, Library, University Archives, Toronto, ONT (contact Sharon P. Larade)

Papers of Harry Lambert Welsh, 1910-1984. Physicist (molecular spectroscopy). Administrative files, correspondence and articles relating to his activities as head of the Department of Physics and his research in molecular spectroscopy.
Stanford University, Green Library, Department of Special Collections and University Archives, Stanford, CA (contact Roxanne Nilan).

Papers of Russell Varian and Sigurd Varian. Co-inventors, with William W. Hansen, of the klystron and rhumbatron and co-founders of Varian Associates. The papers span almost a hundred years, from early manuscripts of poetry by their Irish father, John Varian, to the creation and growth of Varian Associates. In addition to original research notebooks and correspondence regarding the klystron, the collection includes materials on numerous other inventions and ideas from Russell's work for Philo Farnsworth and Humble Oil as well as the brothers' years at Stanford and at Varian Associates. Unpublished short stories by Sigurd reveal his sense of humor as well as his career as a pilot in Mexico and Central America. Ca. 1892-1980.

University of California at Santa Barbara, Library, Department of Special Collections, Santa Barbara, CA (contact Rosanne M. Barker).

Papers of Herbert Phillip Broida, 1920-1978. Physicist (molecular spectroscopy). Contains correspondence, course notes, department notes, letters of reference, post-doctoral applications and appointments, conference and meeting attendee lists, class notes, and purchase orders. Subjects include Western Spectroscopy Association, American Physical Society, National Bureau of Standards, Russian travel, Brazil, Academic Senate, faculty legislature, Astronomy Committee, McGraw-Hill, Memorial fund, and National Aeronautics and Space Administration. Ca. $8 \mathrm{ln}$. ft.

University of California at Santa Cruz, University Library, Mary Lea Shane Archives of the Lick Observa-

tory, Santa Cruz, CA (contact Dorothy Schaumberg). Memorabilia of James Lick, 1796-1876. Books, pictures, newspaper clippings and other memorabilia from the collection of Paquita Lick Machris, great grandniece of James Lick.

Memorabilia of Richard Hawley Tucker, 1859-1952. Photograph albums and memorabilia from the collection of Jane $S$. Tucker, daughter of Richard $\mathbf{H}$. Tucker.

University of Maryland, McKeldin Library, Special Collections Division, Historical Manuscripts and Archives Department, College Park, MD (contact Lauren $R$. Brown).

Papers of Elliott Waters Montroll, 1916-1983. Mathematician and physicist (statistical mechanics, probability, mathematical physics). Collection (bulk dates 1972-1982) contains correspondence, course materials, photographs, publications, reports, reprints, studies, working papers, grant records, and appointment books. 1933-1983. $10.5 \mathrm{ln}$. ft.

Reprinted from AIP History of Physics

Newsletter XIX, No. 2, Dec. 1987 